\title{
Fast neutron and $\gamma$-ray backscatter radiography for the characterization of corrosion-born defects in oil pipelines
}

\author{
Mauro Licata*, Helen M. O. Parker, Michael D. Aspinall, Manuel Bandala, Frank Cave, Sebastian Conway, Domas \\ Gerta and Malcolm J. Joyce \\ * Lancaster University \\ m.licata@lancaster.ac.uk
}

\begin{abstract}
In this research, a combined fast-neutron $/ \gamma$-ray backscatter imaging technique is described. The aim of this work is to understand corrosion defects in pipelines by measuring differences in the scattered radiation flux, generated when different steel thicknesses are irradiated by a neutron and $\gamma$-ray focused beam. A californium-252 radiation source is used to produce fast neutron and $\gamma$ rays, exploiting its spontaneous fission. This mixed radiation field is collimated and directed towards the steel samples. Backscattered neutrons and $\gamma$ rays are measured as a function of the steel thickness using 4 liquid organic scintillation detectors linked to a real-time, pulse-shape discrimination system, which separates and retains the neutron and $\gamma$-ray event data. In this paper, we describe how, using a single radiation source and detection system, it is possible to perform and combine two complementary imaging modalities. This research is validated by an MCNP6 computer simulation study. The backscatter imaging system developed for this research and the experimental results of the measurements carried out using the National Physical Laboratory neutron lowscatter facility are also presented in this paper.
\end{abstract}

Keywords - Fast Neutron tomography, combined neutron- $\gamma$ imaging, backscatter, scintillation detectors, corrosion, pipelines.

\section{INTRODUCTION}

$\mathrm{B}$ ACKSCATTER radiography and backscatter computer tomography (BCT) are non-destructive testing (NDT) techniques that exploit the scattered radiation in contrast to that which is transmitted. The latter is used in the more common and well-known computerized axial tomography (CT). X-ray backscatter imaging has been studied for the last three decades and utilizes the scattered radiation arising by virtue of the Compton Effect, the extent of which depends on the electron density in the scatterer rendering this technique particularly effective for heavy metals. The common procedure for BCT is to probe the object under scrutiny with a collimated pencilbeam and to detect the back-scattered radiation. The main advantage of this particular NDT technique is the investigation of large objects that cannot be scanned with the more usual axial

This work was supported by Innovate UK and coordinated by Hybrid Instruments Ltd.. M. Licata, H. Parker, M. Aspinall, M. Bandala and Malcolm J. Joyce are with the Engineering Department of Lancaster University. F. Cave,
CT approach and furthermore, this technique helps to interpret misleading transmission images that are more difficult to comprehend. X-ray BCT has applications in non-destructive testing particularly for quality control and for security inspection, such as the detection of dangerous materials, border inspections, biomedical science, several engineering fields, above all, oil and gas sector and aerospace. Conversely, there are few if any papers in the literature reporting the use of fast neutrons in place of X-ray as the probing radiation for backscatter radiography. Fast neutrons interact differently compared to $\mathrm{X}$ - and $\gamma$ rays and, in particular, elastic nuclear scattering of neutrons and the corresponding cross section can be exploited, as neutrons have high penetration capabilities, which allow high-density materials to be investigated.

Elastic neutron scattering is a phenomenon exploited for measurements of hydrogen (usually as part of water) content in rocks as well as density and porosity measurements for oil exploration, and for the measurements of the level/presence of water or gas in pipelines. In this research, a simultaneous, fast neutron and $\gamma$-ray backscattered radiography system will be described. Both an extensive Monte Carlo simulation study, computed with the tool MCNP6 [1], and experimental results will be presented. In this particular MCNP6 study, the entire device has been simulated. Such a system will be applied to investigate and characterize defects and possibly corrosion in the walls of oil pipelines, both for the case of no insulation and under insulation (e.g., concrete or polyethylene etc.). Pipelines are subject to several types of corrosion, for example as a consequence of the action of chemical agents, high pressure gases and vapors, crude oil, hydrogen sulfide, and especially water and carbon dioxide. The quantification of corrosion-born defects is, therefore, a key issue in the industry, particularly for all the companies that rely on thousands of kilometres of pipes for transporting their feedstock. Nowadays, ultrasound is the most widespread technology used in pipeline inspections, via inspection gauges deployed inside pipes.

S. Conway and D. Gerta are with Hybrid Instruments Ltd. Corresponding author contact: m.joyce@lancaster.ac.uk 
However, despite the advantage of having high resolution and the possibility of performing measurements quickly, it has the disadvantages that it requires a coupling medium and that it only allows measurements of internal corrosion and not corrosion under insulation. The technique we present is, to some extent, complementary to ultrasound since it does not need a coupling medium; rather it probes the pipes from the outside and has the potential to measure corrosion under insulation.

\section{EXPERIMENTAL METHODS}

Different steel thicknesses have been reproduced and covered with 1-cm layer of two different types of insulation: concrete and polyethylene. The neutron and $\gamma$-radiation field produced by a californium-252 $\left({ }^{252} \mathrm{Cf}\right.$, emission rate: $8.727 \times 10^{6}$ neutrons/second) source has been collimated by means of different layers of lead and high-density polyethylene drilled with 1-cm diameter pinhole in such a way as to produce a pencil-like beam to be focused on the steel surface, as shown in fig. $1 \mathrm{a}$ and $\mathrm{b}$.

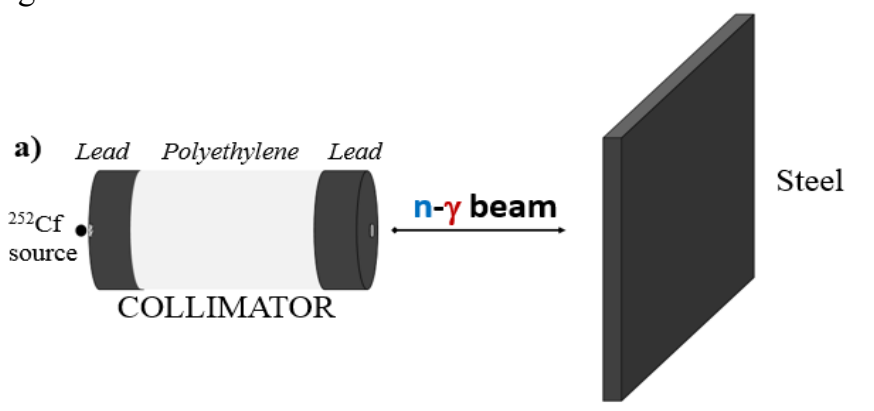

b)

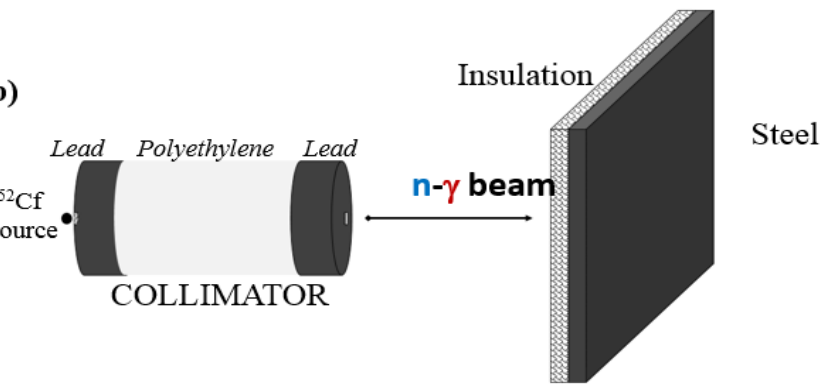

Fig. 1. Illustration of the collimator and the steel slab with (a) and without (b) the presence of insulation.

The presence of defects in the steel, or the manifestation of corrosion and rust, produces a variation of the mean density and a variation of the steel thickness itself, therefore changing the atomic number density, on which elastic scattering by neutrons is heavily dependent. These changes produce a different transmitted-reflected neutrons flux ratio. Scattered fast neutron and $\gamma$-ray fluxes have been measured with an array of EJ-301 (Scionix, Netherlands) scintillation detectors, as a function of the different steel thicknesses. The whole system developed, shown schematically in Fig. 2, comprises a mechanical rig with the aforementioned collimator and detectors. The rig is an aluminum frame of dimensions $600 \times$ $400 \times 340 \mathrm{~mm}$, mounted with two stepper motors configured to move the sub-system comprising the source-collimatordetectors in the X-Y plane to allow the scan of different thicknesses of material. These motors are controlled and driven by an Arduino ${ }^{\circledR}$ board that receives coordinates from the user.

The optimum collimator and detector position were studied extensively via the Monte Carlo tool MCNP6. The geometry and scintillator location were selected in such a way as to have similar collimation factor of fast neutrons and $\gamma$ rays, to maximize the flux on the steel surface and to isolate the detector radiation-sensitive volume from the beam. With this particular configuration, the scintillators are capable of measuring only the backscattered radiation flux generated by the beam focus point. The detectors are placed in a cylindrical assembly using adjustable arms. Such a configuration can accommodate up to 8 detectors, however, in the measurements described in this conference, only 4 detectors were used, since this arrangement maximizes the total geometrical efficiency. The scintillators were connected to a 4-channel mixed radiation field analyzer [2] (MFA, Hybrid Instruments Ltd., UK) capable of providing real-time, pulse-shape discrimination via pulse gradient analysis [3] (PGA), thus retaining separate datasets of both neutrons and $\gamma$ rays. The MFA can generate two transistortransistor-logic (TTL) signals per channel, depending on whether a neutron or a photon has been detected. TTL signals are redirected to an Intel Cyclone V FPGA / ARM Processor system, in which a 16-channel counter has been configured. A program written in $\mathrm{C} \#$ for a PC coupled with a Graphical User Interface (GUI) is capable of monitoring the counters, coordinate and synchronize the collimator-detector position with the respective measured counting rate.

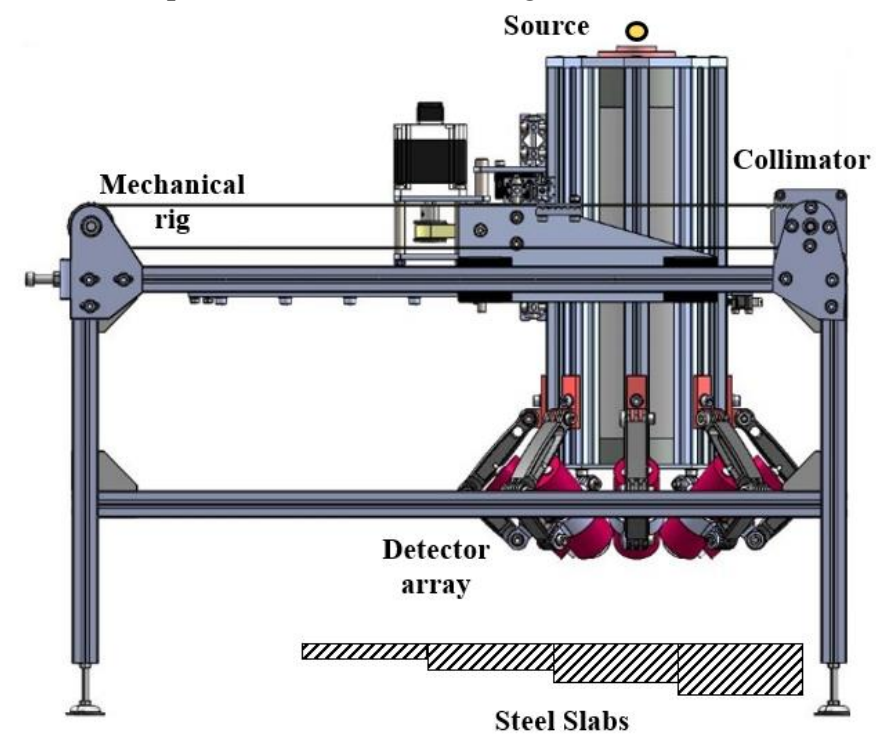

Fig. 2. Schematic, elevation (X-Z plane) of the mechanical rig and the system source-collimator-detectors.

Experiments were carried out at the National Physical Laboratory low-scatter neutron metrology facility (London, UK), in order to minimize the background level. Moreover, a wide range of bare neutron sources of different activities is available at this facility. 

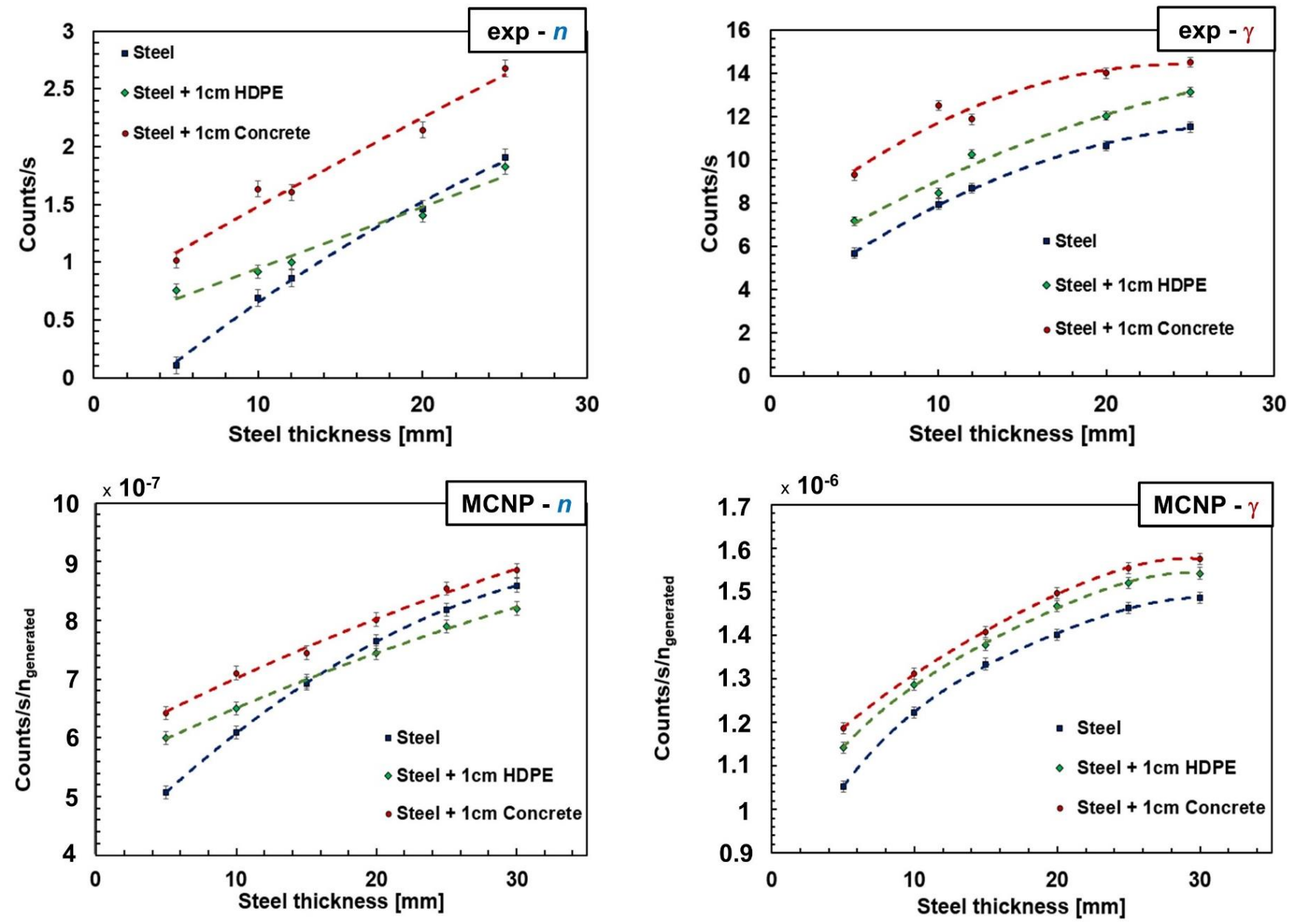

Fig. 3. Top: experimental results of the neutron (left) and $\gamma$-ray (right) backscattered flux for bare steel, steel and polyethylene insulation, and steel and concrete insulation. Bottom: MCNP6 Monte Carlo simulation results of backscatter flux, as a function of the steel thickness, for bare steel, steel and polyethylene insulation, and steel and concrete insulation [5].

\section{RESUlTS}

Reflected neutrons and $\gamma$ rays were detected over a period of 20 minutes for every single slab. The measurement was carried out three times respectively with: bare steel, steel and polyethylene, steel and concrete. The experimental results and the MCNP6 Monte Carlo simulations are shown in Fig. 3. Results are presented with an uncertain of $\pm 1 \sigma$ standard deviation and a second-order polynomial function was chosen to fit the data. Qualitative agreement between experimental results and computer simulations is observed and different steel thicknesses can be clearly discriminated with each other in the range $5-25 \mathrm{~mm}$. As expected, the backscattered flux increases as the steel thickness increases, for both neutrons and $\gamma$ rays, reaching a saturation plateau for the latter, after $20-25 \mathrm{~mm}$. The results also show a difference in the backscatter counting rate as well as a difference in slope for the case of 1-cm layer of polyethylene and concrete. These measurements can be taken as a basis to develop an imaging technique, for instance, radiography or tomography, since the measured backscattered counting rate can be converted, as per analogy with the common transmission imaging, in an image contrast index. With this technique, both neutrons and $\gamma$ rays can be exploited simultaneously and merged with each other, as done in previous research $[4,5]$ for transmission tomography.

\section{CONCLUSION}

In this research, neutron and $\gamma$-ray backscatter are exploited to discern different steel thicknesses. The potential of combining different imaging modalities is also described, providing a valid alternative to the well-known technique of $\mathrm{X}$ ray transmission imaging as well as to the ultrasound technology techniques. Furthermore, an advantage of the system described in this research, is the possibility of performing two different imaging modalities using the same source and detection system. The methods presented in this work have the potential to be applied in several fields. In particular, the use of a radiation source (neutron generators can also be used) opens the system for potential in-situ NDTs and 
applications, for instance, in safety assessments, quality controls and security inspections.

\section{ACKNOWLEDGMENTS}

The authors thank the National Physical Laboratory for the use of the low scatter facility, in particular N. Roberts and G. Taylor for the help during the experimental campaign and with the use of the radiation sources. The authors wish to acknowledge: Lancaster University, the Engineering and Physical Sciences Research Council (EPRSC), the Next Generation Nuclear Centre for Doctoral Training (NGN-CDT), the Lloyd's Register Foundation and Innovate UK, for supporting this project. M. J. J. acknowledges the Royal Society for the award of a Wolfson Research Merit Award.

\section{REFERENCES}

[1] Los Alamos National Laboratory. Los Alamos, NM, USA. https://mcnp.lanl.gov/

[2] Joyce, M. J. et al. The design build and test of a digital analyzer for mixed radiation fields. IEEE. Trans. Nucl. Sci. 57, 2625-2630 (2010).

[3] D'Mellow, B. et al. Digital discrimination of neutrons and $\gamma$-rays in liquid scintillators using pulse gradient analysis. Nuclear Instruments and Methods in Physics Research. Section A. Vol. 578, no. 1, pp. 191-197.

[4] Licata, M. and Joyce M. Concealed nuclear material identification via combined fast-neutron/ $\gamma$-ray computed tomography (FNGCT): a Monte Carlo study. Journal of Instrumentation, Vol. 13, Feb. 2018.

[5] Mauro Licata, Helen M. O. Parker, Michael D. Aspinall, Manuel Bandala, Frank Cave, Sebastian Conway, Domas Gerta and Malcolm J. Joyce. Depicting corrosion-born defects in pipelines with combined neutron / r ray backscatter: a biomimetic approach. Under review, Scientific Reports, March 2019. 